\title{
Relación entre modelo PIHEM y gestión educativa en instituciones educativas del nivel inicial en el distrito de Abancay Apurímac Perú, 2014
}

\author{
Wilber Jiménez Mendoza ${ }^{1}$ \\ Carolina Soto Carrión ${ }^{2}$ \\ Rafael Urrutia Huamán ${ }^{3}$
}

\begin{abstract}
Resumen
Objetivo. Analizar el modelo PIHEM, de gestión de la calidad en la educación, y su relación con la gestión educativa en instituciones educativas del nivel inicial del distrito de Abancay.
\end{abstract}

Método. Se utilizaron instrumentos de recolección de información validados.

Resultados. La gestión educativa hoy se encuentra en un momento dinámico y en medio de debates fundamentales, es indispensable aplicar todos los medios posibles y aprovechar a favor de la educación todo el potencial de todos y cada uno de los agentes que intervienen.

Palabras clave: gestión, liderazgo administrativo, modelo de gestión, agente educativo.

\footnotetext{
1. Doctor en Administración, Magister en Investigación y Docencia Universitaria, Magister en Gestión Pública, Master en Administración Pública y Licenciado en Ciencias de la Comunicación. Profesor Principal a Tiempo Completo de la Universidad Nacional Micaela Bastidas de Apurímac.

Correo: wjimenezmendoza@yahoo.es

ORCID: http://orcid.org/0000-0002-1103-6872

2. Doctora en Medio Ambiente y Desarrollo Sostenible, Magister en Investigación y Docencia Universitaria. Profesora Principal a Tiempo Completo de la Universidad Tecnológica de los Andes de Apurímac. Directora de la Oficina de Relaciones Internacionales.

3. Bachiller en Ciencias de la Educación Universidad Nacional Micaela Bastidas de Apurímac, Licenciado en Educación Física y Danzas (UNAMBA), Licenciado en Educación Inicial (UTEA), Magister en Administración de la Educación por la Universidad César Vallejo de Trujillo, Doctor en Administración de la Educación Universidad César Vallejo de Trujillo.
} 


\title{
Relationship between the PIHEM model and educational management in pre-school institutions in the district of Abancay Apurimac Peru, 2014
}

\begin{abstract}
Objective. Analyze the Pihem model of quality management in education, and its relationship with the management of education in educational institutions of the district's initial level of Abancay.

Method. Collection instruments validated information are used.

Results. Educational management today is in a dynamic moment in the midst of fundamental debates, it is essential to use all possible means and leverage in favor of education the full potential of each and every one of the actors involved.
\end{abstract}

Keywords: Management, administrative leadership, management model, educational agent.

Recibido: 09-04-2015

Aceptado: 29-05-2015

\section{Introducción}

Las significativas transformaciones sociales, económicas, políticas y culturales vividas por la humanidad en el pasado y presente siglo le han asignado un peso cada vez más importante a los factores vinculados con el conocimiento. Estas transformaciones sociales han planteado cambios significativos a los retos del sistema educativo. En estas condiciones se hace necesario "volver a pensar en la educación", sus propósitos, sus contenidos y sus criterios de evaluación. Por otra parte, ante los relativos avances en cobertura, aparece con insistencia la preocupación en torno a las condiciones de calidad en las cuales se desenvuelve el proceso educativo. La amplia participación en los escenarios políticos y sociales contemporáneos, ha conducido a unos crecientes niveles de descentralización y autonomía educativa que exigen una significativa reestructuración de las funciones y responsabilidades, en cada uno de los niveles y nuevas formas de 
control social, sobre el trabajo adelantado en las instituciones educativas; en estas condiciones, se hace necesario reconceptualizar la función del sector (De Zubiría, 2000).

De acuerdo con Casassus (2000), cuando manifiesta que lograr una gestión institucional educativa eficaz, es uno de los grandes desafíos que deben enfrentar las estructuras administrativas para abrir caminos y facilitar vías de desarrollo a un verdadero cambio educativo. Sobre todo, si se entiende a la gestión como una herramienta para crecer en eficiencia, eficacia, pertinencia y relevancia.

Es preciso mencionar además, que la gestión educativa pasa por un enfoque de confusiones, la escuela debe dedicarse a desarrollar el uso del conocimiento, el cultivo de actitudes interpersonales de convivencia equitativa y tener como eje de la acción educativa el aprendizaje, es decir promover la construcción del propio aprendizaje y no la enseñanza, hay también quienes identifican la gestión como administración, hasta considerarlo sinónimos; de acuerdo con Chuye (2007); gestión es una tarea compleja, se conceptualiza como un proceso que articula las múltiples instancias de la vida escolar con una direccionalidad, la cual es la de lograr aprendizajes significativos, gestionar significa enhebrar, hilvanar, entretejer todo aquello que acontece diariamente con miras a lograr un fin. Por otro lado el manual de gestión para directores 2011, publicado por el Ministerio de Educación dice que gestión es la capacidad de articular los recursos de manera que se logren los objetivos, del mismo modo Casassus (2000) afirma que la gestión educativa no es una disciplina teórica, es una disciplina aplicada en la cotidianidad de su práctica por tanto la gestión es una disciplina en la cual interactúan los planos de la teoría, la política y la práctica.

Tanto a nivel regional y local se carece de un modelo que permita evaluar la calidad de la gestión educativa, de modo que facilite y coadyuve a la retroalimentación oportuna de la gestión continua. Por tanto el objetivo fundamental de la presente investigación es determinar la relación entre el modelo PIHEM y gestión educativa en instituciones educativas del nivel inicial en el distrito de Abancay. El capital humano (director, personal docente y administrativo), son la fuerza laboral de toda institución educativa, la cual debe estar bien capacitada y motivada ya que a la larga, el éxito o el fracaso de la organización va a depender de aquellos y no de las restricciones impuestas por problemas sociales, políticos, económicos o medio ambientales.

\section{Modelo PIHEM: modelo peruano}

Es un modelo de gestión de calidad de la educación, que da énfasis a las personas y tiene un marco de referencia en el modelo EFQM, conducente a evaluar la gestión de la calidad administrativa, a nivel personal e institucional, aplicando 
sus cinco principios de calidad en cada uno de sus siete factores y dos factores reservados a los padres de familia y alumnado.

\section{Principios del modelo PIHEM}

Planear: consiste en definir la misión institucional, identificando las funciones y actividades específicas para llevar a cabo la misión. Se identifica a los clientes $\mathrm{y}$ sus necesidades que se traducen en funciones operativas estableciendo indicadores de medición, culminando con el diseño de un plan de acción.

Implementar: poner en práctica en sus dos modalidades, por un lado auto capacitarse o capacitar al equipo líder de calidad, por otro proporcionando los medios, materiales e instrumentos que requiere el modelo.

Hacer: proceso que consiste en ejecutar el plan en forma operativa y las actividades pedagógicas - administrativas.

Evaluar: monitorear los indicadores de medición de las actividades y procesos verificándolos con los clientes.

Mejorar: es el proceso de actuar con el propósito de mejorar continuamente. A partir de aquí se cierra o se abre nuevamente el ciclo.

\section{Fundamentos del modelo PIHEM}

\section{Aspectos generales}

El presente modelo, parte de una visión global de los diferentes componentes de la organización educativa, lo que obliga a tener en cuenta: sus esencias, opiniones, valores y aptitudes. Desde esta perspectiva la evaluación es concebida como un proceso de comprensión y valoración de los procesos y de los resultados; el evaluador es un cooperador con los participantes. En este sentido Stake desarrolla, a mediados de los años 70, una alternativa evaluativa que rompe con la tradición Tyleriana. Este modelo subraya marcos para el desarrollo del aprendizaje, las transacciones didácticas, los datos para los juicios, el informe holístico y la motivación a los educandos es importantísimo. El fin de este tipo de evaluación es ayudar a comprender a profesores, alumnos, personal administrativo, problemas, virtudes y defectos de los programas educativos. Para ello el evaluador debe hacer referencia a las opiniones de la gente sin juzgarlas, recopilando información a lo largo de todo el proceso y dando respuesta a esas cuestiones que se le planteen, para ello se requiere una planificación abierta y flexible tendiendo más a la descripción que a la valoración (Gonzales, 2001:170).

La metodología incluirá la observación-diagnóstica, la planificación estratégica, introducción de técnicas, instrumentos, muestreos intencionales de evaluación, 
informes narrativos. La comunicación con los clientes (profesores y alumnos) ocurrirá de manera natural y permanente.

Conceptual: al referirnos al capital intangible, estamos involucrando a la gestión del conocimiento, a la gestión del capital intelectual, capital social, capital cultural y al capital moral, los mismos que van a reflejar en el desarrollo cognitivo y humano de los agentes de la educación, especialmente, del director, docentes y personal administrativo, los mismos que permanentemente utilizarán diversas modalidades de aprendizaje para generar, incorporar, analizar, almacenar y transferir socialmente los saberes y conocimientos significativos que les permitan potenciar a los discentes en el desarrollo de sus capacidades funcionales y productivas en un mundo de cambio continuo que es la única constante. También hacemos notar, que los desafíos que enfrenta la educación en el mundo y en el Perú son enormes. Entonces la educación de calidad adquiere alta prioridad en el desarrollo de los países, sociedades y organizaciones educativas.

La calidad de la educación que exige el siglo XXI, está centrada en la importante demanda y exige el capital humano con capacidad de administrar un tipo de colegio diferente, considerando al hombre - maestro, como creador y gestor de información y conocimiento; como ser humano centro de la institución, dando énfasis en la calidad y en el desarrollo holístico de las personas y organizaciones, las mismas que se oxigenan a través de diversas modalidades de comunicaciones formales e informales, en donde el estilo de liderazgo participativo tenga como basamento el respeto por los demás, de modo que generen un modelo de gestión de calidad, tendientes a satisfacer las necesidades de los clientes, asociados con las características intrínsecas del servicio.

La calidad no es una característica exclusiva de un servicio, tampoco es un concepto estático, ni tiene fundamento en el costo del servicio, sino es el beneficio o la utilidad que satisface la necesidad de una persona al hacer uso de un servicio o producto; sobre todo, calidad es el resultado de un proceso de gestión integral que abarca todas las etapas de proceso aprendizaje-enseñanza para llegar a producir un buen servicio. En los términos más simples, calidad es un camino no un destino.

Social: la implementación de un modelo de gestión holístico fundamentado en el capital intangible, para mejorar la calidad educativa en una organización educativa, socialmente se fundamenta en garantizar en apoyo de la dirección en diseñar un camino hacia la calidad, haciendo que todas las personas trabajen a favor de la organización educativa previa, innovación y perfeccionamiento que tienda a la satisfacción social de sus integrantes, las mismas que son reflejadas en menores quejas y conflictos con los clientes; óptimo uso de recursos; mejoría de clima laboral; aumento de satisfacción laboral; menor rotación de empleados; aumento de productividad y competitividad; mejoría de la imagen y prestigio de la organización educativa. 
Socialmente se da énfasis en las personas, en una concepción del ser humano, respaldado en la confianza, la honestidad, la ética, la eficiencia, la responsabilidad y la efectividad. Teóricamente se sustentan en la jerarquía de necesidades de Abraham Maslow, la teoría "X" "Y" de Douglas McGregor y la teoría " $Z$ " de Ouchi, que se unen al fundamento práctico a través de sus áreas de capital intangible que eslabonan el aseguramiento de gestión de calidad sostenido por Edwards Deming.

Administrativo: la gestión holística de calidad de un sistema de administración de una institución educativa, se fundamenta en el principio de hacer las cosas bien, en donde es importante la efectividad de un líder para dirigir la misión y visión de la organización centrada en satisfacer las necesidades de los usuarios o clientes.

El cliente, es una persona que tiene una necesidad y para satisfacerla adquiere un servicio o producto. Los clientes (profesores y alumnos) son importantes, pues si no hubiera personas con necesidades o gustos por servicios específicos, no habría demanda, y la organización no tendría razón para existir.

El capital intangible, influye enormemente en la gestión educativa, especialmente cuando se precisa que la calidad no es un problema aislado, sino abarca a toda la institución educativa. El cliente externo (alumno), es lo más importante; los clientes internos (profesor y personal administrativo), son determinantes en los resultados de la gestión; la satisfacción de las necesidades del cliente externo gobiernan todos los indicadores importantes del proceso enseñanza-aprendizaje; la colaboración y el trabajo en equipo son esenciales en el desarrollo de la gestión de calidad; la comunicación efectiva determina eficiencia y éxito y la preocupación principal ahora es encontrar soluciones y no errores, por lo que podríamos concluir que este fundamento de gestión de calidad es un modelo de gestión intensivo en las personas (capital intangible) más no en el capital tangible.

Psicopedagógico: en un modelo de gestión holística, nos impulsa a recurrir a nuevas estrategias para conseguir mejores resultados y que éstas dependen en gran medida de un conocimiento más integral del ser humano. Este es el principio de las organizaciones modernas. En efecto, los cambios más positivos que podamos hacer en nuestras vidas tienen que ver primero con el hecho de llegar a conocernos mejor a nosotros mismos. Como condición necesaria para poder llegar a conocer más y entender mejor a los demás. En gran medida el conocimiento de nosotros mismos comienza por una preocupación en el desarrollo emocional que Daniel Goleman, llama en su libro La inteligencia emocional, la misma que expande la frontera de posibilidades del ser humano en todo sentido, tanto en relación del individuo consigo mismo en sus relaciones de familia, en el trabajo y la sociedad. 
La inteligencia emocional será la inteligencia del tercer milenio, porque se requerirá mayor tolerancia, mejor convivencia, las mismas que pueden ser aprendidas. Y que desde esta perspectiva las instituciones educativas que hasta ahora han ignorado el tema enfrentarán en el futuro próximo y es esta disciplina, uno de los mayores desafíos de responsabilidades y una oportunidad pendiente. También se fundamenta psicopedagógicamente en las teorías: genética de Piaget, cognitiva de Ausubel, histórica-cultural de Vigotsky y humanista de Roger.

\section{Factores del modelo PIHEM}

Son las partes integrantes del conocimiento gerencial global, que agrupa características, cualidades propias de la institución educativa y su relación con el entorno. Estas características adquieren sentido e identidad en la medida que integran y fortalecen los procesos formativos que se gestan y se desarrollan en las dimensiones administrativas y pedagógicas: comprende siete factores.

La elección del Modelo PIHEM se sustenta en:

Justificación Legal: La gestión educativa está entrelazada de forma inseparable con la dirección de las instituciones educativas, en los aspectos institucional, administrativo, pedagógico y comunitario, pero a su vez está regulada por reglas, leyes, normas o dispositivos. Las reglas proporcionan ciertas características a la gestión de la institución educativa y determinan en alguna medida el accionar de los directores.

Justificación Organizacional: Antúnez y Gairín (2000), consideran a la dirección como el órgano vital de la institución educativa, ya que en torno a ella giran decisiones sobre la organización y funcionamiento de la escuela, de ella depende la vinculación con la comunidad y otras instituciones; su función es básica por influir en las conductas del personal en beneficio de la educación de los alumnos.

Justificación Epistemológica: "La gestión educativa eficaz, ética, descentralizada y con participación de la ciudadanía" es objetivo estratégico de las políticas educativas al 2021 y como resultado se espera lograr "fortalecer una participación social responsable y de calidad en la formulación, gestión y vigilancia de las políticas y proyectos educativos", hecho que se trasunta en la generación, o adaptación de nuevos conocimientos a una realidad concreta.

Justificación metodológica: El desarrollo del presente estudio, de acuerdo al diseño de investigación elegido, permitirá establecer la relación entre el modelo PIHEM con la gestión educativa, contribuyendo los resultados a verificar si la gestión de las instituciones educativas del ámbito de estudio, cumplen con las funciones pedagógicas, administrativas y financieras como son, hacer seguimiento, evaluar las metas y objetivos de los planes y programas de estudio y sus estrategias para su implementación, relacionado en organizar, orientar, 
observar las instancias de trabajo técnico-pedagógico y de desarrollo profesional de los docentes, finalmente promover un adecuado clima organizacional en la institución educativa y en lo relacionado a asignar, administrar y controlar los recursos.

\section{Metodología}

Se utilizó un diseño no experimental, de carácter descriptivo correlacional y transversal, (Hernández Sampieri, 1998), porque en un primer momento se describe y analiza dos fenómenos en forma independiente mediante el estudio del mismo en una circunstancia de tiempo-espacio determinado, para luego medir el grado de relación y/o asociación que existe entre dichos conceptos o variables.

\section{Población y muestra}

\section{Población}

La población objeto de estudio estará conformada por todas las instituciones educativas del nivel inicial del distrito de Abancay.

\section{Muestra}

Para definir el tamaño de la muestra se utilizó la técnica del Muestreo No Probabilístico de carácter Intencionado, por las mismas características de las variables en estudio.

\section{Resultados}

\section{Variable: modelo PIHEM}

A los directores pertenecientes a la muestra seleccionada se les aplicó cuatro instrumentos correspondientes a medir cada una de las dimensiones.

La variable "Modelo PIHEM", permitió conocer cuatro aspectos importantes: liderazgo para la calidad, planificación estratégica, satisfacción de clientes, calidad, impacto social y medio ambiente, Tablas 1 y 2. 
Tabla 1. Escala de calificación para la variable: modelo PIHEM.

\begin{tabular}{|c|l|c|l|}
\multicolumn{2}{|c|}{$\begin{array}{l}\text { Escala } \\
\text { Para dimensiones }\end{array}$} & \multicolumn{2}{|c|}{$\begin{array}{l}\text { Escala } \\
\text { Para puntaje total }\end{array}$} \\
\hline $0-1$ & EXISTE PLAN & $0-4$ & EXISTE PLAN \\
\hline $1-2$ & $\begin{array}{l}\text { SE HA IMPLEMENTADO EL } \\
\text { PLAN }\end{array}$ & $4-9$ & $\begin{array}{l}\text { SE HA IMPLEMENTADO EL } \\
\text { PLAN }\end{array}$ \\
\hline $2-3$ & $\begin{array}{l}\text { PLAN REALIZADO SIN } \\
\text { EVALUAR }\end{array}$ & $9-13$ & $\begin{array}{l}\text { PLAN REALIZADO SIN } \\
\text { EVALUAR }\end{array}$ \\
\hline $3-4$ & $\begin{array}{l}\text { PLAN REALIZADO Y } \\
\text { EVALUADO }\end{array}$ & $13-17$ & $\begin{array}{l}\text { PLAN REALIZADO Y } \\
\text { EVALUADO }\end{array}$ \\
\hline $4-5$ & $\begin{array}{l}\text { PLAN REALIZADO Y MEJORA } \\
\text { COTINUA }\end{array}$ & $17-20$ & $\begin{array}{l}\text { PLAN REALIZADO Y MEJORA } \\
\text { COTINUA }\end{array}$ \\
\hline
\end{tabular}

Fuente: Elaboración propia.

Tabla 2. Estadísticos descriptivos para la dimensión: liderazgo para la calidad.

\begin{tabular}{|c|c|c|c|c|c|}
\hline & & Frecuencia & Porcentaje & $\begin{array}{c}\text { Porcentaje } \\
\text { válido }\end{array}$ & $\begin{array}{c}\text { Porcentaje } \\
\text { acumulado }\end{array}$ \\
\hline \multirow{3}{*}{ Válidos } & Se ha implementado el plan & 1 & 1,2 & 1,3 & 1,3 \\
\cline { 2 - 6 } & Plan realizado sin evaluar & 11 & 12,8 & 13,8 & 15,0 \\
\cline { 2 - 6 } & Plan realizado y evaluado & 12 & 14,0 & 15,0 & 30,0 \\
\cline { 2 - 6 } & Plan realizado y mejora continua & 56 & 65,1 & 70,0 & 100,0 \\
\cline { 2 - 6 } & Total & 80 & 93,0 & 100,0 & \\
\hline Perdidos & Sistema & 6 & 7,0 & & \\
\hline Total & & 86 & 100,0 & & \\
\hline
\end{tabular}

Fuente: SPSS 21.

En la Tabla 2, se observa que un $65,1 \%$ de sujetos de la muestra de estudio, consideran que la dimensión liderazgo para la calidad se encuentra en un rango de plan realizado y mejora continua, mientras que un $14 \%$ considera en un rango de plan realizado y evaluado respecto a la dimensión liderazgo para la calidad. 
Wilber Jiménez Mendoza, Carolina Soto Carrión, Rafael Urrutia Huamán

Relación entre modelo PIHEM y gestión educativa en instituciones educativas del nivel inicial en el distrito

de Abancay Apurímac Perú, 2014. Artículo producto de la investigación.

Tabla 3. Estadísticos descriptivos para la dimensión: planificación estratégica.

\begin{tabular}{|c|c|c|c|c|c|}
\hline & & Frecuencia & Porcentaje & $\begin{array}{c}\text { Porcentaje } \\
\text { válido }\end{array}$ & $\begin{array}{c}\text { Porcentaje } \\
\text { acumulado }\end{array}$ \\
\hline \multirow{4}{*}{ Válidos } & Existe plan & 5 & 5,8 & 6,3 & 6,3 \\
\cline { 2 - 6 } & Se ha implementado el plan & 34 & 39,5 & 42,5 & 48,8 \\
\cline { 2 - 6 } & Plan realizado sin evaluar & 33 & 38,4 & 41,3 & 90,0 \\
\cline { 2 - 6 } & Plan realizado y evaluado & 7 & 8,1 & 8,8 & 98,8 \\
\cline { 2 - 6 } & Plan realizado y mejora continua & 1 & 1,2 & 1,3 & 100,0 \\
\cline { 2 - 6 } & Total & 80 & 93,0 & 100,0 & \\
\hline Perdidos & Sistema & 6 & 7,0 & & \\
\hline Total & & 86 & 100,0 & & \\
\hline
\end{tabular}

Fuente: SPSS 21.

En la Tabla 3, se observa que un 39,5\% de sujetos de la muestra de estudio, consideran que la dimensión planificación estratégica, se encuentra en un rango de se ha implementado el plan, mientras que un 38,4\% considera en un rango de plan realizado sin evaluar, respecto a la dimensión liderazgo para la calidad.

Tabla 4. Estadísticos descriptivos para la variable: modelo PIHEM.

\begin{tabular}{|c|c|c|c|c|c|}
\hline & & Frecuencia & Porcentaje & $\begin{array}{c}\text { Porcentaje } \\
\text { válido }\end{array}$ & $\begin{array}{c}\text { Porcentaje } \\
\text { acumulado }\end{array}$ \\
\hline \multirow{4}{*}{ Válidos } & Existe plan & 3 & 3,5 & 3,8 & 3,8 \\
\cline { 2 - 6 } & Se ha implementado el plan & 12 & 14,0 & 15,0 & 18,8 \\
\cline { 2 - 6 } & Plan realizado sin evaluar & 32 & 37,2 & 40,0 & 58,8 \\
\cline { 2 - 6 } & Plan realizado y evaluado & 28 & 32,6 & 35,0 & 93,8 \\
\cline { 2 - 6 } & Plan realizado y mejora continua & 5 & 5,8 & 6,3 & 100,0 \\
\cline { 2 - 6 } & Total & 80 & 93,0 & 100,0 & \\
\hline Perdidos & Sistema & 6 & 7,0 & & \\
\hline Total & & 86 & 100,0 & & \\
\hline
\end{tabular}

Fuente: SPSS 21.

En la Tabla 4, se observa que un $37,2 \%$ de sujetos de la muestra de estudio, consideran que la variable modelo PIHEM, se encuentra en un rango de plan realizado sin evaluar, mientras que un $32,6 \%$ lo considera en un rango de plan realizado y evaluado. 


\section{Variable: gestión educativa}

Para la variable "gestión educativa", se consideró el instrumento de recopilación de datos, el mismo que contiene un total de veinte preguntas distribuidas en sus cuatro dimensiones: gestión institucional, gestión administrativa, gestión pedagógica y gestión comunitaria; cada una de ellas con cinco ítems o preguntas respectivamente. Los puntajes obtenidos por cada uno de los sujetos encuestados, se presentan a continuación, Tabla 5.

Tabla 5. Escala de calificación para la variable: gestión educativa.

\begin{tabular}{|l|l|c|l|}
\hline \multicolumn{2}{|c|}{$\begin{array}{c}\text { Escala } \\
\text { Para dimensiones }\end{array}$} & $0-4$ & \multicolumn{2}{c|}{$\begin{array}{c}\text { Escala } \\
\text { Para puntaje total }\end{array}$} \\
\hline $0-1$ & DEFICIENTE (D) & $4-9$ & MALO (M) \\
\hline $1-2$ & MALO (M) & $9-13$ & REGULAR (R) \\
\hline $2-3$ & REGULAR (R) & $13-17$ & BUENO (B) \\
\hline $3-4$ & BUENO (B) & $17-20$ & EFICIENTE (E) \\
\hline $4-5$ & EFICIENTE (E) & & \\
\hline
\end{tabular}

Fuente: Elaboración propia.

\section{Relación entre dimensiones de las variables modelo PIHEM y gestión educativa}

A continuación se presentan los resultados obtenidos respecto a las correlaciones que se dan entre la variable modelo PIHEM y sus dimensiones liderazgo para la calidad; planificación estratégica; satisfacción de los clientes; y calidad, impacto social y medio ambiente con la variable gestión educativa con sus dimensiones gestión institucional; gestión administrativa; gestión pedagógica y gestión comunitaria, Tabla 6 . 
Tabla 6. Tabla de contingencia entre dimensiones liderazgo para la calidad y gestión institucional.

\begin{tabular}{|c|c|c|c|c|c|}
\hline & & \multicolumn{4}{|c|}{ Gestión institucional } \\
\hline & & Malo & Regular & Bueno & Total \\
\hline \multirow{4}{*}{$\begin{array}{l}\text { Liderazgo para la } \\
\text { calidad }\end{array}$} & $\begin{array}{c}\text { Se ha implementado } \\
\text { el plan }\end{array}$ & 1 & 0 & 0 & 1 \\
\hline & $\begin{array}{c}\text { Plan realizado sin } \\
\text { evaluar }\end{array}$ & 7 & 4 & 0 & 11 \\
\hline & $\begin{array}{c}\text { Plan realizado y } \\
\text { evaluado }\end{array}$ & 0 & 7 & 5 & 12 \\
\hline & $\begin{array}{l}\text { Plan realizado y mejora } \\
\text { continua }\end{array}$ & 3 & 26 & 27 & 56 \\
\hline Total & & 11 & 37 & 32 & 80 \\
\hline
\end{tabular}

Fuente: SPSS 21

\section{Discusión}

Con respecto al modelo PIHEM, variable 1, Tabla 6 , que un $37,2 \%$ de los docentes considera que dicha variable se encuentra en un rango de plan realzado sin evaluar, mostrando tendencias positivas, donde algunas comparaciones externas visualizan situaciones satisfactorias; mientras que un $32,6 \%$ lo considera en un rango de plan realizado y evaluado, la misma que nos muestra tendencias muy positivas dentro del modelo de gestión y tan solo un 5,8\% de docentes considera u rango de plan realizado y mejora continua; significando esto que este porcentaje menor considera que sus instituciones donde laboran muestran una insistencia de mejoramiento continuo de calidad manteniendo una posición de liderazgo.

Para la variable gestión educativa, un 38,4\% de los docentes considera que dicha variable se encuentra en un rango de regular, mientras que un 31,4\% lo considera en un rango de bueno y tan solo un 4,7\% lo califica como eficiente a la gestión educativa. Sin embargo es imprescindible señalar que siendo la educación un proceso tan importante, se necesita que la gestión dentro de las instituciones educativas del nivel inicial alcancen mayores puntajes, mostrándonos que hay una tendencia a mejorar por parte de los agentes educativos y así lograr que la gestión educativa sea cada vez mejor.

En lo referente a la relación entre las dimensiones liderazgo para la calidad y gestión institucional, existe un correlación de Pearson de 0,512 que muestra una significativa correlación entre ambas dimensiones, lo cual nos indica que el factor liderazgo ejercida tanto por el director como por los profesores en las instituciones del nivel inicial guarda relación respecto a la gestión institucional 
propiamente dicha creando un prestigio y a la vez una buena imagen ante la sociedad.

Con respecto a la relación entre las dimensiones planificación estratégica y gestión administrativa, existe una correlación de Pearson de 0,589 que muestra una correlación significativa entre ambas dimensiones, lo cual nos indica que la planificación estratégica está bien encaminada en las instituciones del nivel inicial y a su vez esto repercute de manera positiva en la gestión administrativa, logrando cada vez más consolidar sus capacidades para planear, implementar, hacer, mejorar y evaluar.

Con respecto a la relación entre las dimensiones satisfacción del usuario y gestión pedagógica, existe una correlación de Pearson de 0,661 que muestra una correlación significativa entre ambas dimensiones, lo cual nos indica que el usuario quien recibe el servicio educativo muestra satisfacción por la gestión pedagógica brindada en las instituciones del nivel inicial en el distrito de Abancay.

Con respecto a la relación entre las dimensiones calidad, impacto social y medio ambiente y gestión comunitaria, existe una correlación de Pearson de 0,640 que muestra una correlación significativa entre ambas dimensiones, lo cual nos indica que las instituciones del nivel inicial en el distrito de Abancay están tomando en cuenta dentro de sus documentos de planificación el trabajo direccionado hacia la comunidad y la población tomando como punto de partida la problemática local referente al cuidado del medio ambiente así mismo poniendo en práctica el reciclaje.

Finalmente los resultados para la correlación entre la variables modelo PIHEM y gestión educativa, presentán un coeficiente de correlación de Pearson que toma el valor de 0,750 , con una significatividad del 0,01 , lo cual nos indica que para los puntajes totales de estas variables existe una correlación importante, puesto que el modelo PIHEM está orientado a evaluar la gestión administrativa y esta a su vez es parte de la gestión educativa encontrándose semejanzas y correlaciones en los datos recogidos de los cuestionarios aplicados. 


\section{Referencias bibliográficas}

Alvarado, O. 1999. Gestión educativa: enfoques y procesos. Lima: Fondo de Desarrollo Editorial. Universidad de Lima.

Aguerrondo, I. 2004. La gestión en los años 90. Algunas cuestiones para mejorar la eficacia educativa. Santiago de Chile: UNESCO.

Ander-Egg, E. 1993. La planificación educativa, conceptos, métodos, estrategias y técnicas para educadores. Primera Edición. Buenos Aires: Editorial Magisterio del Río de la Plata.

Antúnez, S. Gairín, J. 2000. La organización escolar. Práctica y fundamentos. Barcelona: Editorial Grao.

Amarante, A. 2000. Gestión Directiva. Módulos 1 a 4. Buenos Aires: Magisterio del Río de la Plata.

Calero, M. 1999. Gestión Educativa: Administración Educativa. Primera Edición. Lima: Ediciones Abedul.

Casassus, J. 2000. Problemas de la gestión educativa en América Latina (la tensión entre los paradigmas de tipo A y el tipo B). Edición $\mathrm{N}^{\circ}$ 01. Santiago de Chile: UNESCO, OREALC.

Casassus, J. 1998. Descentralización de la gestión a las escuelas y calidad de la educación: ¿mitos o realidades? Revista de Tecnología Educativa $N^{o} 01$ Buenos AiresArgentina.

Contrera, B. 2006. Problemas de la gestión educativa en América Latina (La tensión entre los paradigmas de tipo A y el tipo B). Edición $\mathrm{N}^{\circ}$ 01. Santiago de Chile. UNESCO, OREALC.

Chuye, Y. 2007. Introducción a la teoría general de la administración. Quinta Edición. Bogotá: Editorial McGraw Hill.

Chuye, Y. 2007. Participación de los actores de la institución educativa en la gestión del cambio. Lima - Perú.

De Zubiría, M. 2006. Los modelos pedagógicos. Bogotá: Cooperativa editorial del Magisterio.

Gonzales, E. 2001. Necesidades de formación de los directores de escuelas secundarias generales del Estado de Aguas Calientes. México.

Hernández, R. 1998. Metodología de la Investigación. zera. Edición. México: Mc- Graw Hill. 
Murillo, J. 1999. Manual del Director. Lima, 1ra. Edición.

Poyato, J. 2009. La investigación sobre eficacia escolar. Barcelona: Editorial REICE.

Rubio, M. 1999. El Sistema Jurídico (Introducción al Derecho). Lima: Pontificia Universidad Católica del Perú.

Sánchez, H. Reyes, C. 2002. Metodologías y diseños en la investigación científica. Lima: Universidad Ricardo palma. Editorial Universitaria.

Trathemberg, L. 1996. Paradigmas para la Dirección Escolar Moderna. En C. Appenzeller y otros. Gestión descentralizada de la educación. Lima: Coincide.

Valdés, T. 1998. Educar para el siglo XX. En Democracia, desarrollo e integración. Buenos Aires: OEl. Ed. Troquel.

Valiente, P. 2010. La profesionalidad del director escolar: sus competencias fundamentales. La Habana: Edit. Holguín.

Verdugo, O. 1992. La descentralización educativa. Lima: Editorial Asociación de publicaciones educativas. TAREA. 